\title{
High Resolution Images of High Entropy \& Multi-Metallic Nano Particles
}

Alexander Lehr ${ }^{1}$, Miguel Yacaman ${ }^{2}$, Jesus Velazquez Salazar ${ }^{2}$ and John Sanchez ${ }^{2}$

${ }^{1}$ Northern Arizona University, Bellemont, Arizona, United States, ${ }^{2}$ Core faculty Center for Materials Interfaces in Research and Applications (MIRA) - Northern Arizona University, United States

Nano particles have been an area of great interest for several decades due to their emergent optical, magnetic, and other properties. This has fueled the more recent excitement with multi component nanoparticles. These multi component nanoparticles have synergetic effects such as in field like catalysis. Though there are many ways to synthesis such nanoparticles, the rules that govern their growth and formation are less known. It is thought that the emergent properties of nanoparticles are due in part to the higher surface/internal atom ratio, this work sets out to understand the role this plays in the formation of multi-metallic nanoparticles. Due to the small size of the nanoparticles, advanced imaging and analytical techniques are required to understand their structure. Therefore, we are presenting HR-STEM images of the atomic structure of Platinum-Copper metal alloy nanoparticles ( $\mathrm{Pt}-\mathrm{Cu} \mathrm{NP}$ ). The imaging of the $\mathrm{Pt}-\mathrm{Cu}$ NP along with EELS/EDS analytical techniques have allowed us to peer into these nanoparticles to understand the thermal entropy properties that govern both the crystalline structure and the morphology.

In this study, Pt-Cu NP were synthesized by combining thermal reduction and high entropy by the addition of metal alloys. Figure 1 shows HR-STEM images of the structure formed by $\mathrm{Pt}$ and $\mathrm{Cu}$ atoms. The FFT, inset in Figure 1, allows us to infer the direction of the $\mathrm{Cu}$ (200) planes along with the Pt structure. This marks a clear difference between the two crystalline structures of the metal alloy $\mathrm{Pt}-\mathrm{Cu}$ NP, which gives us valuable information into the formation and properties of the nanoparticles. Furthermore, EELS analysis has revealed the presence of $\mathrm{Cu}$ atoms (Figure 2) along a supper lattice structure formed mainly by $\mathrm{Pt}$ atoms. It is possible to correlate the $\mathrm{Pt}-\mathrm{Cu}$ positions in the superlattice by superimposing the brightest spots corresponding to $\mathrm{Pt}$ with the inner ones lying between those $\mathrm{Pt}$ atoms that correspond to $\mathrm{Cu}$.

The Pt-Cu NPs studied here were fabricated using traditional wet chemistry methods. $0.1 \mathrm{ml}$ of $\mathrm{PtCl}$ and $\mathrm{CuCl}$ were prepared with a molarity of $0.1 \mathrm{M}$. They were then mixed with $5 \mathrm{ml}$ of oleylamine and then heated to $250 \mathrm{C}$ for two hours. $0.01 \mathrm{ml}$ of 1-dodecanethiol was then added and heated for an additional hour. The solution went from a golden yellow color to a dark red color and finally a dark brown color. The solution was then cleaned to remove any remaining solvent so that the nano particles could be easily imaged. HR-STEM images allow to determine that $\mathrm{Pt}-\mathrm{Cu}$ particles were on the order of $10 \mathrm{~nm}$ in size.

Understanding the structure of nanoparticles allows for better synthesis and tailoring to desired properties. HR-STEM allowed us to identify crystal structure and this information will be used to form rules on how nanoparticles are formed. 


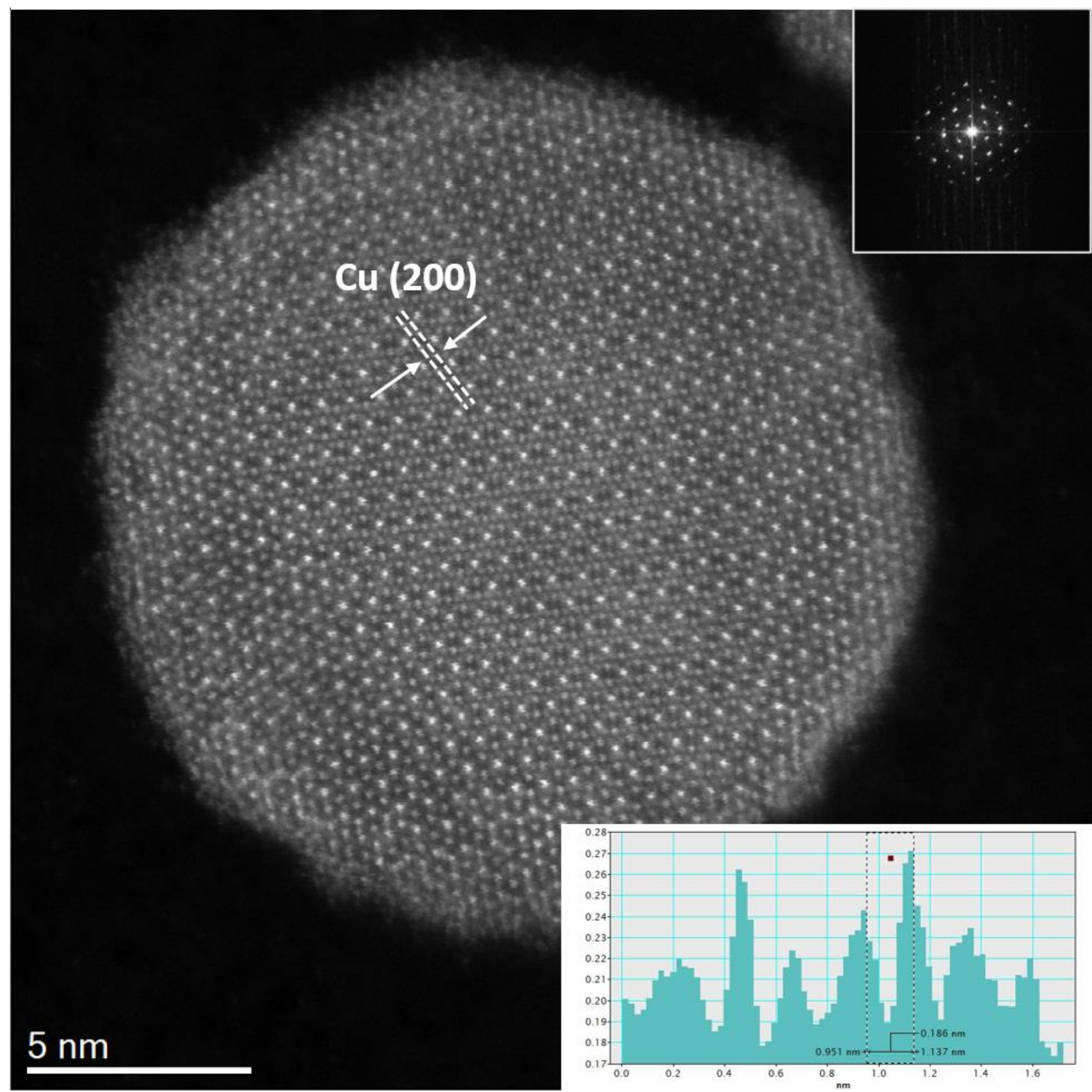

Figure 1. HR-STEM image of Pt-Cu nanoparticle. The upper inset shows the FFT of the image used to index the copper planes. Bottom inset shows distance between planes. It can be seen that there is a superlattice formed mainly by platinum atoms. 

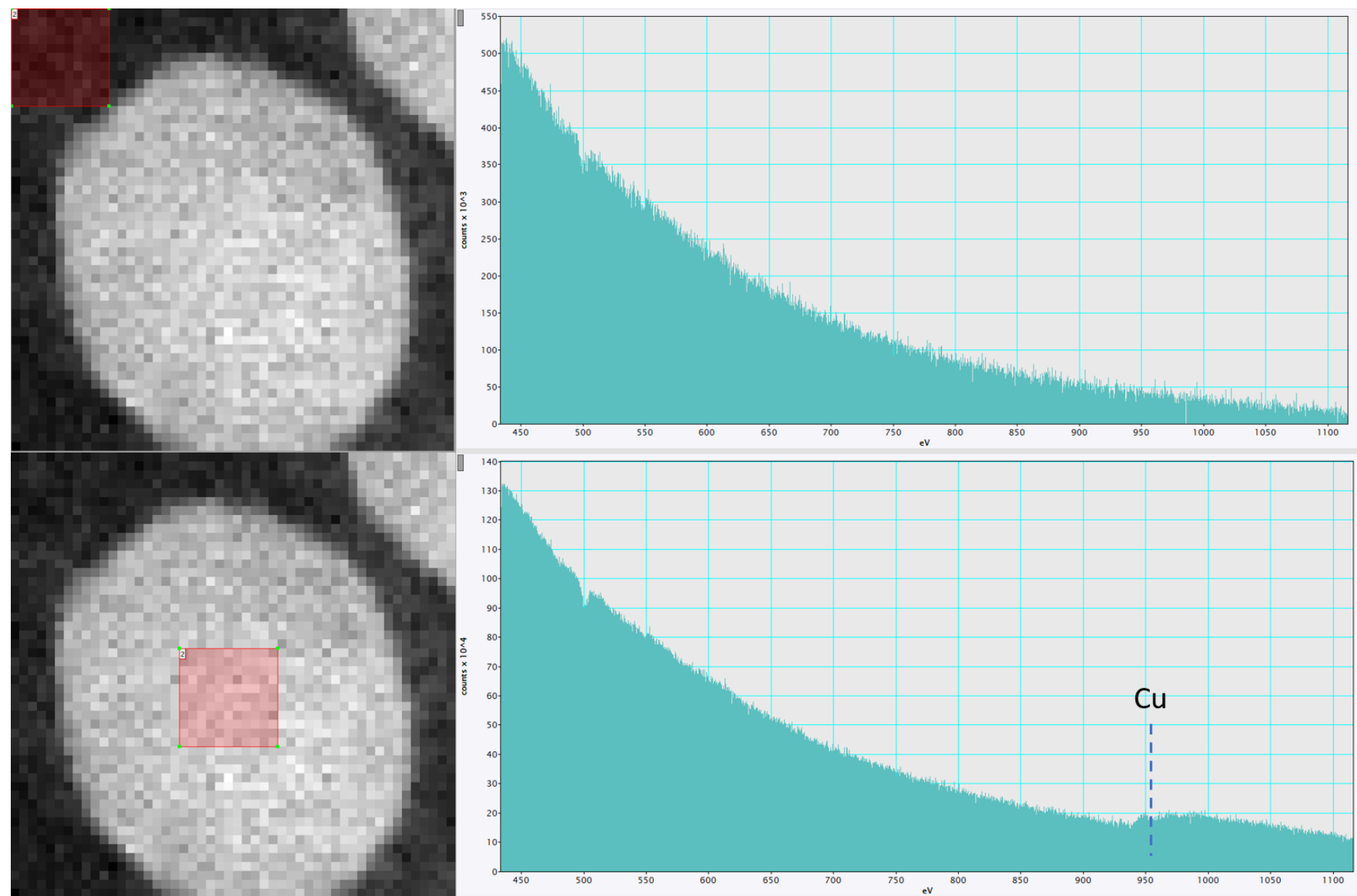

Figure 2. EELS mapping shows energy loss around $951 \mathrm{eV}$ corresponding to the copper atoms.

\section{References}

Guisbiers, G. (2019). Advances in thermodynamic modelling of nanoparticles. Advances in Physics: X, 4(1), 1668299. doi:10.1080/23746149.2019.1668299

Yücelen, Emrah; Lazić, Ivan; Bosch, Eric G. T. . (2018). Phase contrast scanning transmission electron microscopy imaging of light and heavy atoms at the limit of contrast and resolution. Scientific Reports, 8(2676). doi:10.1038/s41598-018-20377-2 